9th AIAA/ASME Joint Thermophysics and Heat Transfer Conference

June 5-8, 2006, San Francisco, CA

\title{
Aerothermodynamic Analyses of Towed Ballutes
}

\author{
Peter A Gnoffo; Greg Buck† James N. Moss; Eric Nielsen§; Karen Berger $₫$ William T. Jones $\|$ \\ NASA Langley Research Center, Hampton, VA 23681-2199 \\ Rena Rudavsky** \\ Columbia University, New York, NY, 10027-6902
}

\begin{abstract}
A ballute (balloon-parachute) is an inflatable, aerodynamic drag device for application to planetary entry vehicles. Two challenging aspects of aerothermal simulation of towed ballutes are considered. The first challenge, simulation of a complete system including inflatable tethers and a trailing toroidal ballute, is addressed using the unstructured-grid, Navier-Stokes solver FUN3D. Auxiliary simulations of a semi-infinite cylinder using the rarefied flow, Direct Simulation Monte Carlo solver, DSV2, provide additional insight into limiting behavior of the aerothermal environment around tethers directly exposed to the free stream. Simulations reveal pressures higher than stagnation and corresponding large heating rates on the tether as it emerges from the spacecraft base flow and passes through the spacecraft bow shock. The footprint of the tether shock on the toroidal ballute is also subject to heating amplification. Design options to accommodate or reduce these environments are discussed. The second challenge addresses time-accurate simulation to detect the onset of unsteady flow interactions as a function of geometry and Reynolds number. Video of unsteady interactions measured in the Langley Aerothermodynamic Laboratory 20-Inch Mach 6 Air Tunnel and CFD simulations using the structured grid, Navier-Stokes solver LAURA are compared for flow over a rigid spacecraft - sting - toroid system. The experimental data provides qualitative information on the amplitude and onset of unsteady motion which is captured in the numerical simulations. The presence of severe unsteady fluid - structure interactions is undesirable and numerical simulation must be able to predict the onset of such motion.
\end{abstract}

\section{Introduction}

Large, inflatable ballutes (balloon-parachutes) have been proposed as hypersonic decelerators for planetary aerocapture applications. ${ }^{1-3}$ The mantra of ballute design is "Fly higher, fly lighter, fly cooler". ${ }^{4}$ Peak heating occurs at much higher altitudes in the transitional flow regime for large ballutes as compared to rigid aerobrakes. Larger braking area means lower convective heating levels. Ballutes may be directly clamped to a vehicle or towed behind the vehicle as a semi-independent device that can be quickly cut free when the requisite change in velocity is achieved. Inflation occurs on the order of hours prior to atmospheric entry. Preliminary estimates ${ }^{4}$ show a favorable decelerator system mass to total mass fraction of $10 \%$. Corresponding estimates for a rigid aeroshell system solution are at least $25 \%$. However, simulation of the aerothermal environment of a spacecraft-ballute system is more complex than a rigid system. Inflatable systems are subject to larger, possibly unsteady, deformations. Ballutes are typically deployed at altitudes requiring analyses that span continuum to non-continuum flow both because of low free stream densities and because of the range of length scales (tether diameter on the order of centimeters to ballute diameters on

\footnotetext{
*Senior Research Engineer, Aerothermodynamics Branch; AIAA Fellow

$\dagger$ Research Engineer, Aerothermodynamics Branch,Senior Member AIAA

${ }^{\ddagger}$ Senior Research Engineer, Aerothermodynamics Branch,AIAA Fellow

$\S$ Research Scientist, Computational AeroSciences Branch,Member AIAA

"Research Engineer, Aerothermodynamics Branch,Member AIAA

"Research Engineer, Advanced Engineering Environments Branch, Member AIAA

** Doctoral student, Columbia University

2006
} 
the order of tens of meters). Uncertainties in non-equilibrium radiative heating levels tend to be higher as thermochemical-nonequilibrium effects become more prominent at higher Knudsen numbers. Perhaps the only simplification of ballute system aerothermal analysis compared to rigid aeroshells is that ballutes, under nominal design conditions, do not ablate.

Aeroheating on spherical and toroidal ballutes has been documented for the limiting case that ignores interactions from the wake of the towing spacecraft. ${ }^{5}$ These studies were implemented to obtain initial estimates of drag coefficient and requisite ballute size for a specific mission given constraints on the surface temperature of the ballute material. They established ballute-only aerodynamics and heating for undeformed shapes across the transitional flow regime from free-molecular to continuum using Direct Simulation Monte Carlo (DSMC) (DAC code ${ }^{6}$ ) and continuum Navier-Stokes (LAURA code ${ }^{7,8}$ ).

Simulations of spacecraft - towed ballute interactions, including spherical and toroidal inflatables, were subsequently reported. These studies did not include the tow lines (tensile tethers or inflatable columns to support compressive loads.) They compared aerothermal environments as a function of trailing distance for the spherical towed ballute. It also investigated the extent of reverse flow through the wake of the spacecraft in the toroidal ballute case as a function of Reynolds number. The occurrence of significant unsteadiness in the wake core was noted as the Reynolds number increased approaching the ballute cut-free point. Prediction of such unsteady reverse flow with increasing Reynolds number is consistent with the limiting inviscid simulation first reported by Hornung. ${ }^{9}$

The objective of this paper is to focus more closely on two challenging aspects of aerothermal simulation of towed ballutes. The first challenge addresses simulation of a complete system, including the tow lines. The long term goal of such a simulation is to include: (1) unsteady surface boundary conditions admitting large scale deformations fully coupled to a structural response analysis, (2) time-accurate simulation of the flow-field, and (3) adaptive physics providing appropriate models for flow analysis as a function of local Knudsen number. The approach used here is to apply an unstructured grid simulation with the expectation that it provides the best opportunity for future spatial, temporal, and physics model adaptation. The unstructured grid solver is FUN3D ${ }^{10}$ using LAURA and VULCAN ${ }^{11}$ derived modules for flux reconstruction and thermochemical non-equilibrium gas models. The current simulation is for a steady flow case with rigid surfaces that include four tow lines connecting the spacecraft to the toroidal ballute. Symmetry boundary conditions at 0 degrees and 90 degrees are applied to reduce computational resource requirements for this initial investigation. The analysis assumes validity of the Navier-Stokes equations; however, an independent analysis of a semi-infinite tether using the DSV2 code provides context from a DSMC perspective.

The second challenge addresses the need to discern unsteady flow interactions using a temporally accurate simulation. The long term goal, as noted previously, is to provide time accurate simulations with quantifiable uncertainties. A short term goal explored here is to identify the onset of large, unsteady interactions impinging on any surface for the purpose of restricting design space (if possible) away from any such unsteady domain. Consequently, it is important for the simulation to admit unsteady flow if it exists and to capture the amplitude of unsteadiness. However, details of the time dependent response are less important. First-order in time iterations are employed for this screening purpose. A flight simulation case near the ballute cut-free time is re-examined in this way using the structured grid, Navier-Stokes solver, LAURA. Ground-based tests of Mach 6 flow over a spacecraft - toroidal ballute - sting system are then simulated in which the onset of unsteady flow is strongly affected by the separation distance between the spacecraft and the toroid. Temporal data acquisition in the experiment was not sufficient to provide code validation quality data for time dependent response but it did capture the onset and extent of unsteady interactions through video of shock motion, serving the objective of this study.

\section{Numerical Tools}

\section{A. LAURA}

Langley Aerothermodynamic Upwind Relaxation Algorithm (LAURA) is a high fidelity, structured grid analysis tool, specialized for hypersonic re-entry physics, utilizing state-of-art algorithms for computational fluid dynamic (CFD) simulations. ${ }^{7,8}$ Key elements of LAURA include Roes averaging ${ }^{12}$ and Yee's Symmetric Total Variation Diminishing (STVD) ${ }^{13}$ formulation of second-order, inviscid flux. Yee's STVD formulation has been found to be exceptionally robust and Courant-number-independent using first point-implicit and then line-implicit relaxation for hypersonic flow simulations. The TVD algorithm uses a non-linear, minmod function as a flux limiter that maintains second-order accuracy away from extrema but can admit limit 
cycles in the convergence process, particularly in the vicinity of captured shocks. This occurrence usually manifests itself as a stalling of convergence at a very low error norm, essentially a benign ringing in the solution at a level that has no impact on aerothermodynamic quantities. Viscous flux is computed using central differences.

The LAURA algorithm, in its default mode, uses single level storage and relaxes the steady form of the governing equations with constant, large Courant number. However, the simulations reported here require time accuracy. Time accurate solutions (first-order in $\Delta t$ ) can be recovered by using constant time-step advancement at all cells and by employing two levels of storage with sub-iterations to converge the current iterate before advancing to the next time level. ${ }^{14,15}$ The inviscid flux, viscous flux, and source terms are evaluated at advanced time level $n+1$ through sub-iterations. The source term for each conservation law now includes the time rate of change of conserved variable $\mathbf{q}$ in volume element $\Omega$ using the first-order accurate, discrete approximation $\left(\mathbf{q}_{L}^{n+1}-\mathbf{q}_{L}^{n}\right) \Omega_{L} / \Delta t$. In theory, sub-iterations continue until $\Delta \mathbf{q}_{L}^{k+1}=\mathbf{q}_{L}^{k+1}-\mathbf{q}_{L}^{k}$ is converged to order $\Delta t^{2}$. In practice $\mathbf{q}_{L}^{n+1}$ is updated after 10 sub-iterations on index k before advancing to time step $n+2$.

\section{B. DS2V}

Heating analyses at the lower densities of transitional rarefied flow require the Direct Simulation Monte Carlo (DSMC) technique developed by Bird. ${ }^{16}$ DSMC simulates gas flows by modeling the motion and collisions of millions of representative molecules based on the kinetic theory of gases. The DSMC technique captures the non-equilibrium in translational and internal degrees of freedom, which is strongly evident in most rarefied flows, and uses phenomenological models to describe the inelastic collisions that may occur between gas molecules and surfaces. The DSMC simulations discussed herein use the DS2V program of Bird. ${ }^{17}$ It is a general 2D/axisymmetric code that provides both time accurate unsteady flow and timeaveraged flow simulations. Molecular collisions are simulated with the variable hard sphere (VHS) molecular model. Energy exchange between the translational and internal modes is controlled by the Larsen-Borgnakke statistical model. ${ }^{18}$

\section{FUN3D}

FUN3D is a node based, fully unstructured, finite volume solver of the Euler and Navier-Stokes equations. ${ }^{10}$ The unstructured grid formulation provides the ability to resolve complex, flexible/deforming geometries. (In the present application, only rigid bodies are considered.) A suite of modules have recently been added to synthesize all of the gas physics models in LAURA and VULCAN ${ }^{11}$ for thermodynamics, transport properties, chemical kinetics, and thermal relaxation. Furthermore, the inviscid flux reconstruction algorithms used in LAURA (Roe, Yee, and Harten algorithms noted previously) have been modified where necessary for an unstructured grid context and incorporated in the generic gas path through FUN3D. ${ }^{19,20}$ FUN3D is still undergoing validation for hypersonic applications - primarily because the quality of heat transfer in the stagnation region of blunt bodies in hypersonic flow often exhibits significant excursions about the mean of about 10 to 20 percent. These excursions are associated with high aspect ratio cells in the boundary layer combined with entropy waviness streaming from inadequately resolved (grid aligned) bow shocks. However, in the lower Reynolds number domain simulated here the observed heating levels appear to exhibit all of the expected symmetry properties.

\section{Spacecraft - Tether - Ballute System Simulation}

\section{A. Continuum Analysis of System}

A simulation of a spacecraft - tether - ballute system using the unstructured grid, Navier-Stokes flow solver FUN3D is presented in Figs. 1 - 8. The ballute has a $52 \mathrm{~m}$ ring diameter and $13 \mathrm{~m}$ cross-sectional diameter. Conditions of the simulation are for a Titan Organics Explorer with velocity equal to $8550 \mathrm{~m} / \mathrm{s}$ and density equal to $1.910^{-7} \mathrm{~kg} / \mathrm{m}^{3}{ }^{1,2}$ All surface temperatures are set to a constant value equal to $500 \mathrm{~K}$. The gas model includes molecular nitrogen and atomic nitrogen in thermochemical nonequilibrium. The towing spacecraft is a Pathfinder shape - $70 \mathrm{deg}$ spherically capped cone with a $6 \mathrm{~m}$ base diameter. The simulation domain encompasses a 90 degree wedge about the system axis. The spacecraft looks sharp at the nose in the figures because of the view angle combined with the 90 degree domain cutting planes. The simulation 
assumes symmetric flow with four $0.3 \mathrm{~m}$ diameter compressive tethers attached to the toroid so that the leading edge of the tether is tangent to the toroid outer surface. A compressive tether is a flexible cylinder which can be inflated to withstand compressive loads and is used to position the toroid in space prior to entry. Tensile load tethers would likely be encased within the compressive tether. At present, only a continuum simulation is enabled. Flow over the compressive tethers is deep in the transitional flow domain in which the validity of Navier-Stokes analyses is inaccurate. Consequently, an independent analysis of the tether using DSMC is discussed in the next section. Ideally, a fully coupled continuum - rarefied analysis would be brought to bear on this complex system of disparate length scales.

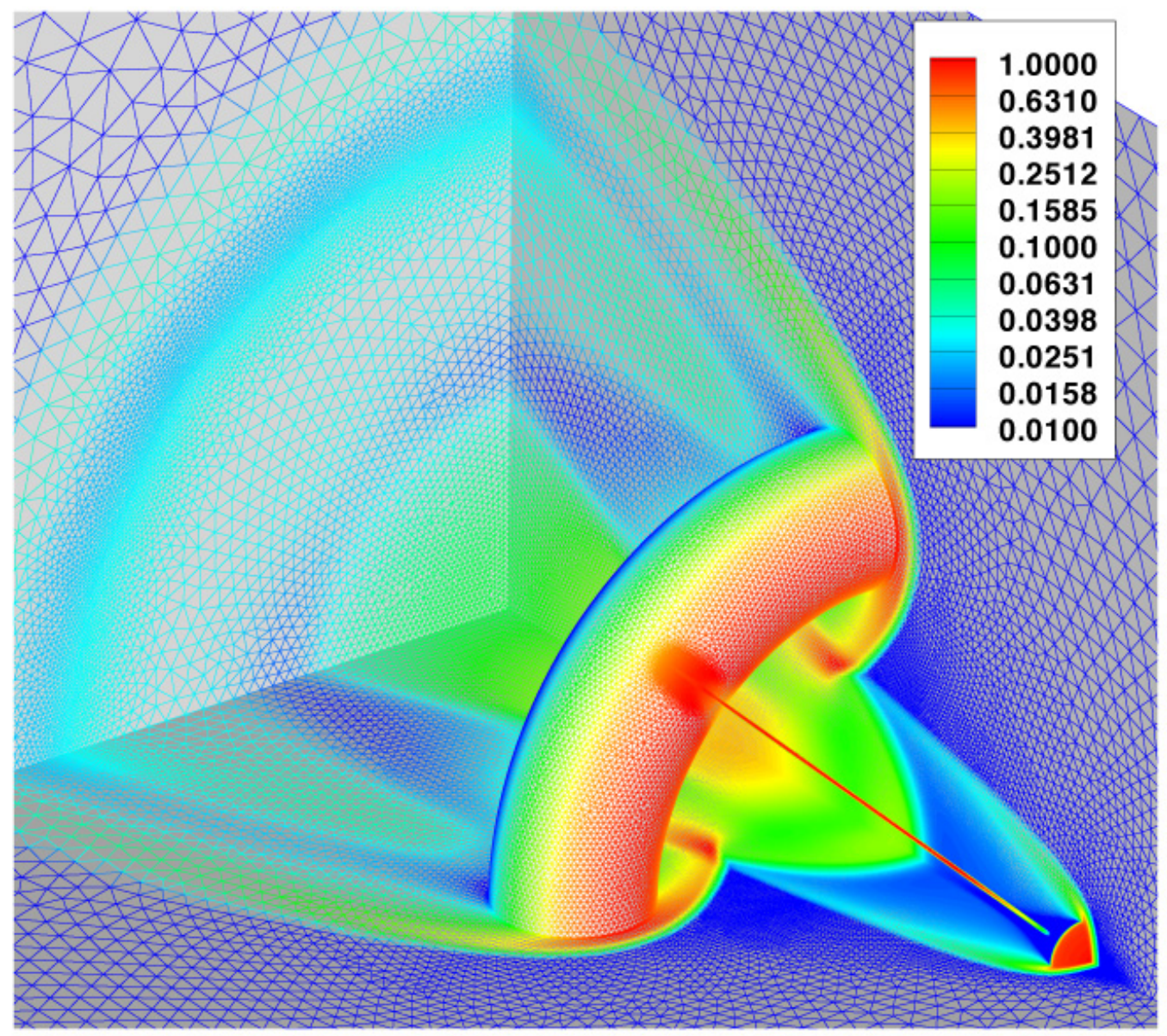

Figure 1. Unstructured grid used in simulation of spacecraft - tether - ballute system. Colors correspond to pressure levels nondimensionalized by $\rho_{\infty} V_{\infty}^{2}$.

The quarter domain in Fig. 1 is discretized with 992,102 nodes. Flow over the spacecraft in Fig. 2 is in a merged layer, transitional regime. The Knudsen number based on spacecraft diameter is approximately 0.057. The boundary layer extends across the entire shock layer to the captured bow shock. There are approximately 20 points across this domain with cell Reynolds number on the order of 0.2 at the surface. Flow over the ballute is still in a transitional regime, but is closer to the continuum domain than the towing spacecraft. The Knudsen number based on ballute cross-sectional diameter is 0.026 . The cell Reynolds number at the ballute surface is approximately 0.2 with approximately 20 nodes extending across the shock layer and 3 to 4 nodes extending across the "inviscid" portion of the shock layer. The largest mesh height stretching factor is approximately 2.0 and it occurs where the advancing front of the boundary domain over the ballute meets the isotropic grid away from the solid surface. Flow conditions over the tether vary according to the location. In the domain where the tether is directly exposed to the free stream the Knudsen number based on tether diameter is approximately 0.88 , placing it near the free molecular limit of the 


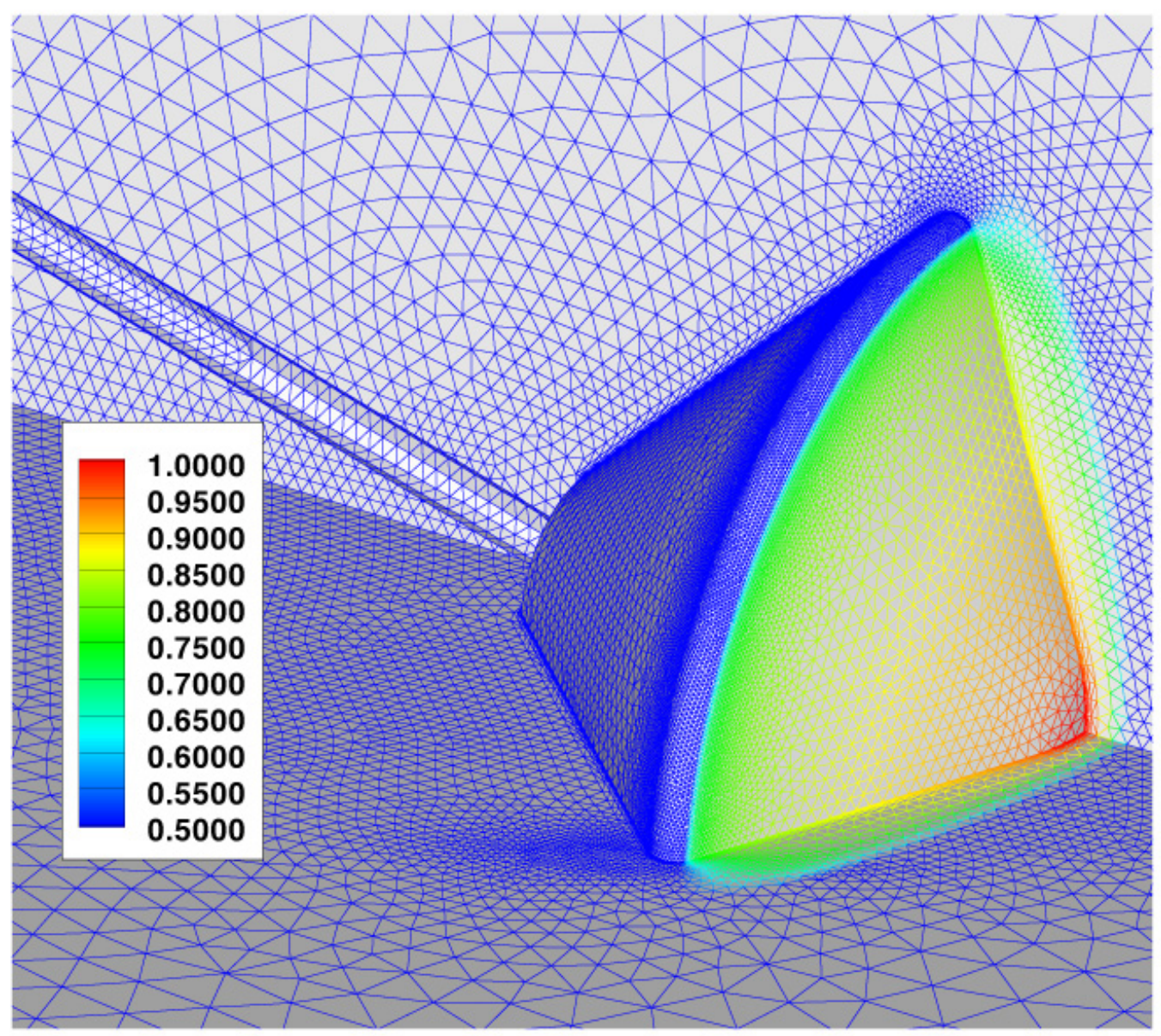

Figure 2. Detail of unstructured grid used surrounding spacecraft. Colors correspond to pressure levels nondimensionalized by $\rho_{\infty} V_{\infty}^{2}$.

transitional flow regime. A spring adaptation algorithm was tested to sharpen the captured shock. In this case, the shock resolution came at the expense of resolution of the shock layer above the boundary layer on the ballute and so results from the original grid were retained. Near wall grid resolution is well within accepted norms for simulation of surface heating. Observed flow structures and surface quantity magnitudes are consistent with previous, structured grid solutions of a related configuration. However, results presented herein with FUN3D should be considered qualitative at present. Stretching factors of two in the merged layer exceed accepted structured grid metrics and there is lack of validation or grid convergence study in this domain. Furthermore, issues remain regarding ability to compute heat transfer with high aspect ratio, tetrahedral cells in the boundary layer within the stagnation region of a blunt body in hypersonic flow. ${ }^{20}$

Pressure distributions in the plane of symmetry away from the tether are presented in Fig. 3. The extent

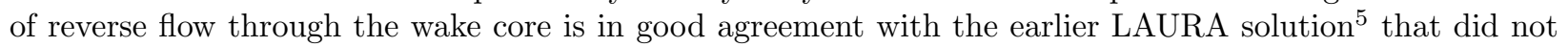
include the tether. The flow is nearly frozen chemically, consistent with the spacecraft Knudsen number, as indicated in Fig. 4. It is not until the flow crosses the ballute bow shock or enters the recirculating flow driven by the converging shocks in the core of the toroid that residence times in a high temperature domain are long enough to enable dissociation. In like manner, thermal nonequilibrium is evident in Fig. 5. Vibrational temperatures lag translational temperatures by nearly $20,000 \mathrm{~K}$ at the shock front but then exceed translational temperature in the recirculating core, 


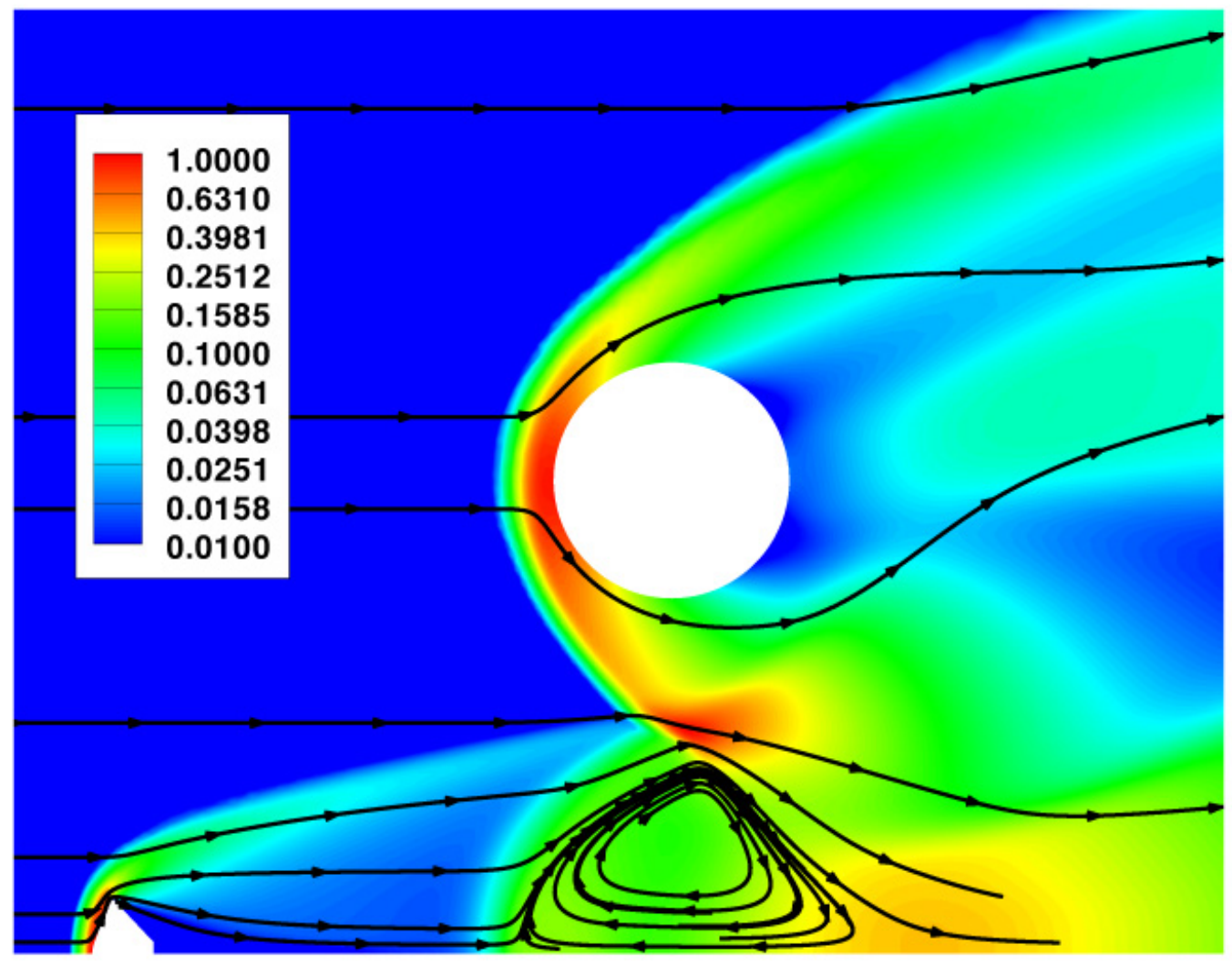

Figure 3. Pressure contours and streamlines in symmetry plane. Colors correspond to pressure levels nondimensionalized by $\rho_{\infty} V_{\infty}^{2}$ 


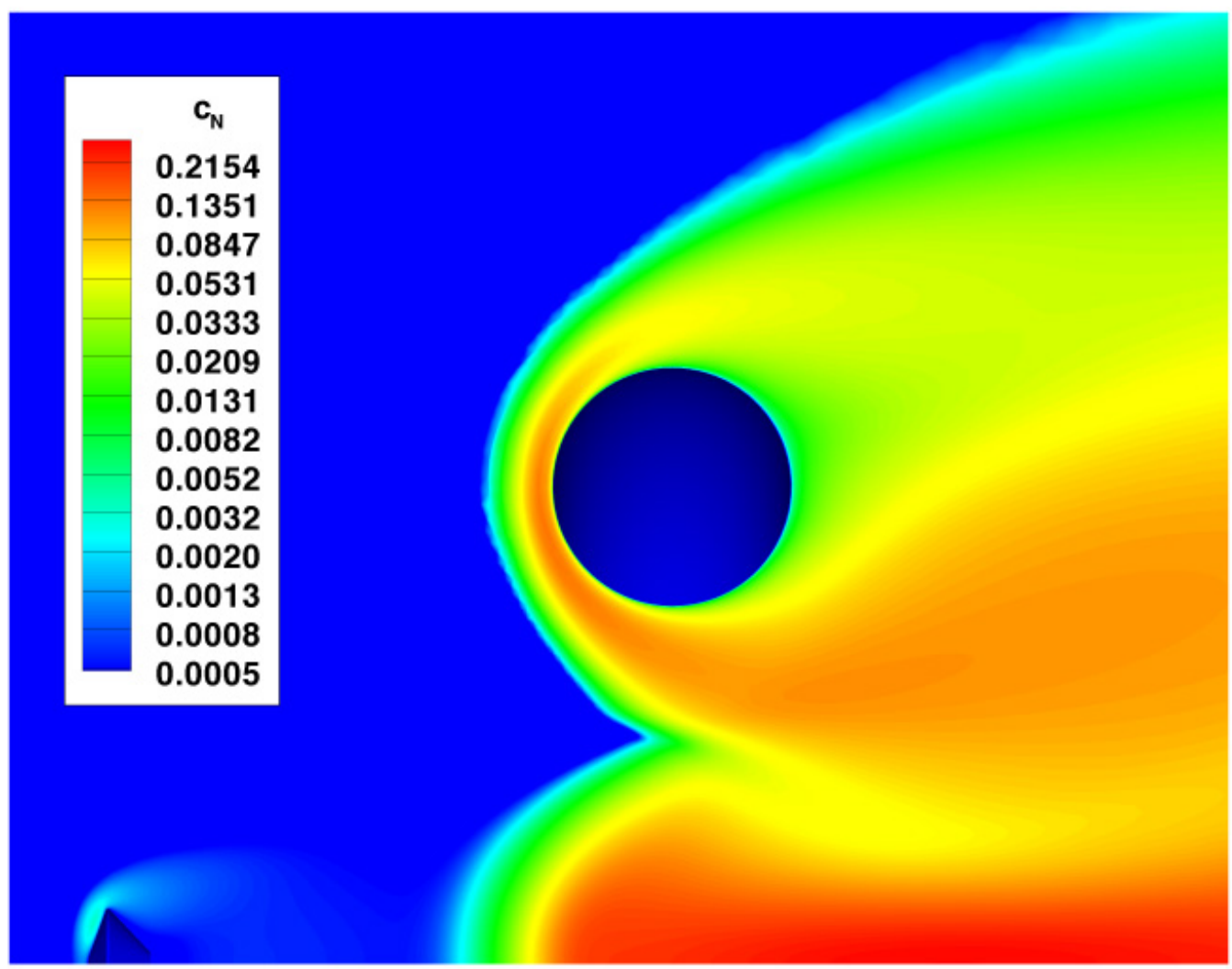

Figure 4. Atomic nitrogen mass fraction contours in symmetry plane.

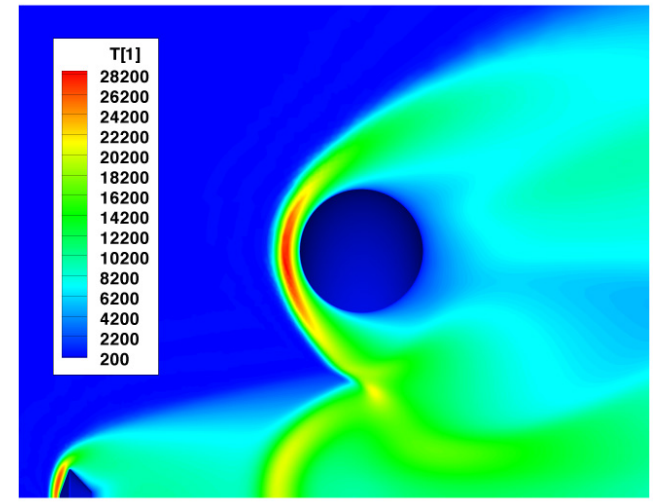

(a) Translational temperature

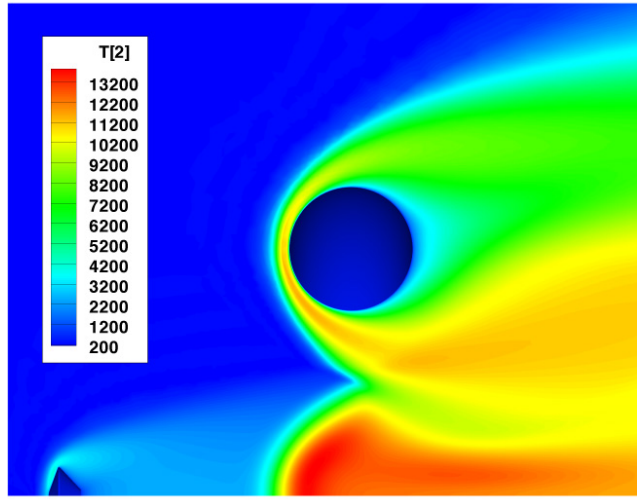

(b) Vibrational temperature

Figure 5. Temperatures in symmetry plane. 


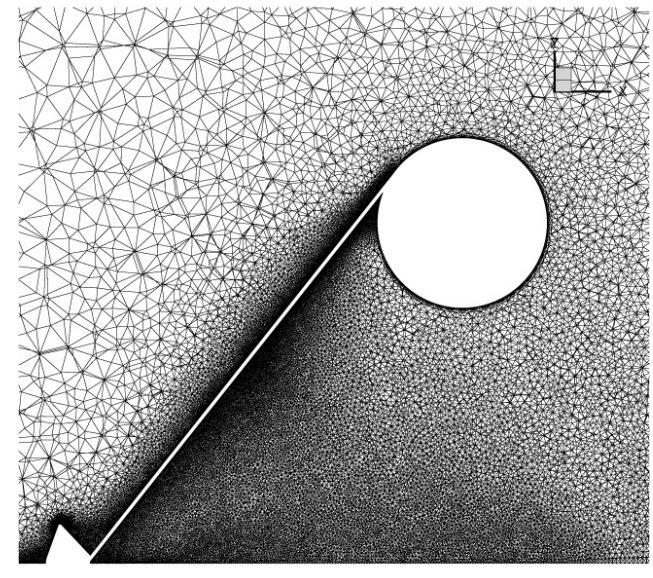

(a) Grid

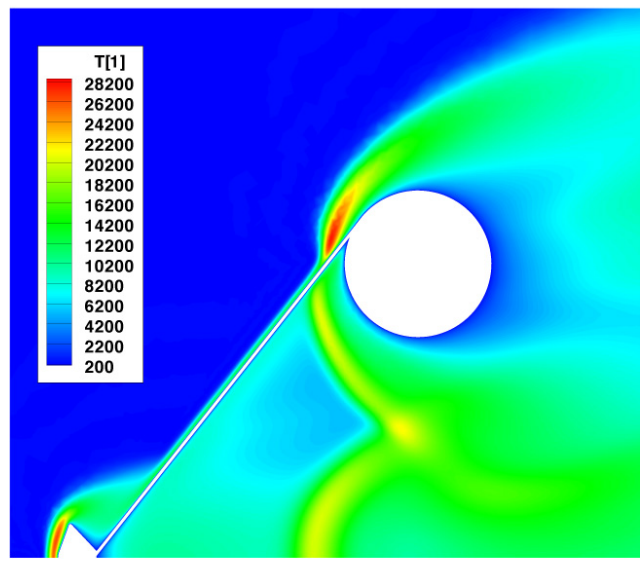

(b) Temperature

Figure 6. Cut plane through tether at 45 degrees.

Rotating the cut plane 45 degrees about the x-axis bisects the compressive tether, as shown in Fig. 6. The tether's wake has insignificant impact on the extent of reverse flow through the spacecraft wake core (compare Fig. 5(a) with Fig. 6(b)). The ballute bow shock is locally offset at the point where the tether cuts through it. The high temperatures above the tether at the ballute attachment point wash across the tether. The effect on local heating is shown in subsequent figures.

A front view of the entire system, including grid, pressure, and heating is presented in Fig. 7. Surface grid in the vicinity of the attachment point is clustered to pick up details of the interaction. A high pressure and heating occurs on the ballute surface associated with the shock over the tether interacting with the bow shock over the ballute as seen in Fig. 7(b) and Fig. 7(c). These interactions are resolved in more detail in Fig. 8. Surface streamlines show that the attachment line is pulled further outboard toward the tether attachment point. The heating rate on the ballute surface is approximately $50 \%$ larger than nominal rates on the attachment line away from the tether. Pressures too are greater than nominal values on the attachment line due to the focusing of the ballute shock - tether shock interaction.

The highest heating and pressure on the tether occur where it emerges from behind the spacecraft and passes through the spacecraft bow shock. A similar phenomenon is observed on the leading edge of the space shuttle wing in hypersonic flight in which the efficient staged compression from the bow shock and wing produce locally high pressures, though less than stagnation levels. In this case, the compression through the spacecraft bow shock followed by the shock formed over the inclined tether produce a large pressure in the interaction. The smaller tether radius serves to amplify the heating in this interaction which is about $20 \%$ greater than nominal heating values on the tether attachment line. (Fig. 8(b))

With benefit of Figs. 6(a), 6(b), and 8(d) it appears that a more benign interaction may be expected if the tether were to be attached from a more outboard location on the spacecraft and connected to the inboard tangency point on the toroid. This setup will reduce the inclination angle of the tether relative to the free stream and will divert the tether wake away from the ballute surface which serve to reduce heating levels. A possible downside of such a configuration is the pinching of the flow between the tether and ballute (and associated heating increase) as it expands towards the wake. 


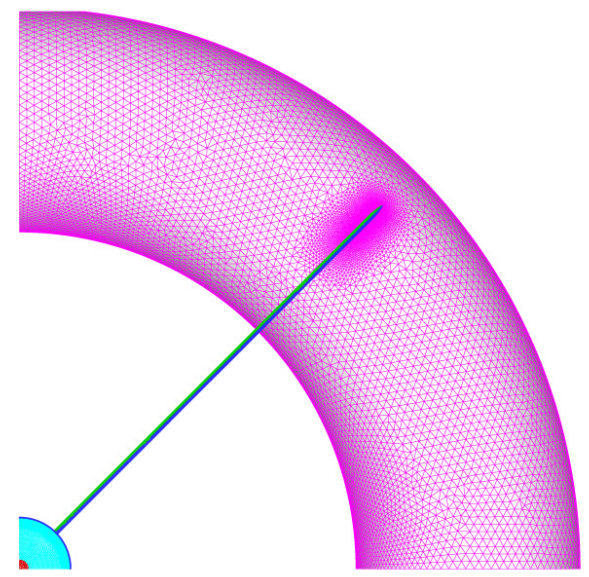

(a) Grid

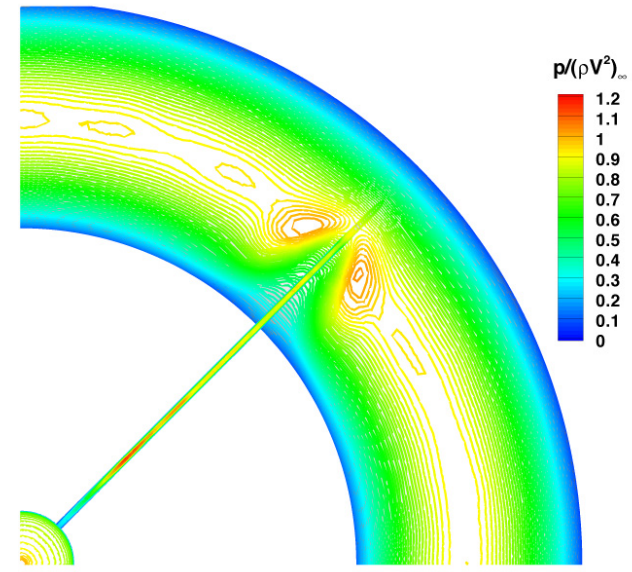

(b) Pressure

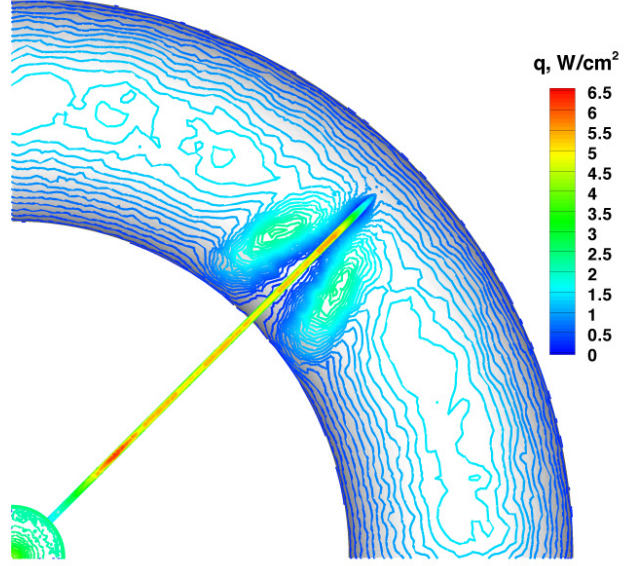

(c) Heating

Figure 7. Front view of surface pressure and heating.

\section{B. Rarefied Analysis of Tether}

A series of DSMC simulations have been executed on an idealized tether, modeled as an infinite yawed cylinder in a uniform hypersonic flow. ${ }^{21}$ The cylinder is inclined to the flow at 45 degrees. Flow conditions correspond to the peak heating trajectory point $\left(V_{\infty}=4750 \mathrm{~m} / \mathrm{s}, \rho_{\infty}=1.710^{-6} \mathrm{~kg} / \mathrm{m}^{3}, T_{\infty}=165 \mathrm{~K}\right)$ for a 5 to 1 toroidal ballute at Titan in a nitrogen atmosphere (presence of methane ignored in this simulation). The surface is assumed to be non-catalytic. These conditions represent an updated mission focus but the trends are instructive in reviewing the variations from large to small Knudsen numbers. The simulations for a $1 \mathrm{~cm}$ and $1 \mathrm{~mm}$ diameter tensile tether (loads in tension only) are presented in Figs. 9 and 10. The simulations for a $50 \mathrm{~cm}$ diameter compressive tether (inflated flexible column accepts loads in compression) are presented in Figs. 11 and 12.

In the case of small diameter tethers in the limit of large Knudsen number (small diameter compared to mean free path) one expects that: (1) incoming particles strike tether without colliding with any reflected particles, and (2) heating rates are independent of tether diameter but strongly dependent on inclination angle. The independence of heating rate is confirmed in Fig. 10 in which stagnation point heating for a 1 $\mathrm{cm}$ diameter tether (left) is very close to the $1 \mathrm{~mm}$ diameter tether (right). The heating rate in both cases is approximately $0.33 \rho V^{3}\left(\approx 5.8 \mathrm{~W} / \mathrm{cm}^{2}\right)$. The radiative equilibrium wall temperature in this case is 1050 $\mathrm{K}(780 \mathrm{C})$, ignoring conduction and re-radiation through leeside of tether. Strong thermal nonequilibrium is clearly evident in that the peak vibrational temperature occurs in the wake of the tether. Note that this 


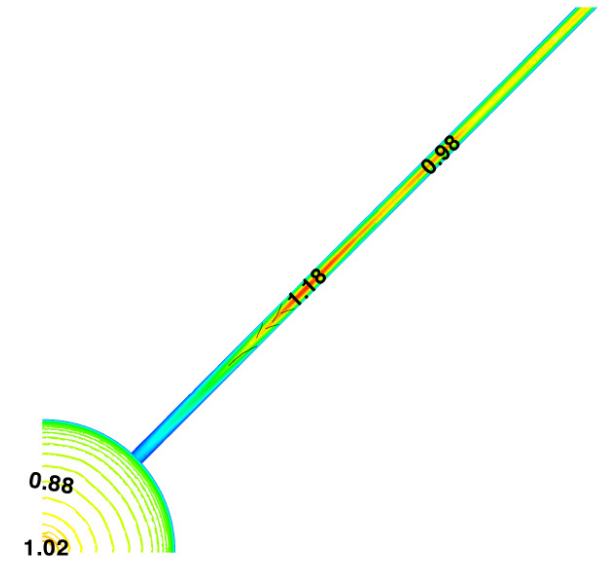

(a) Pressure

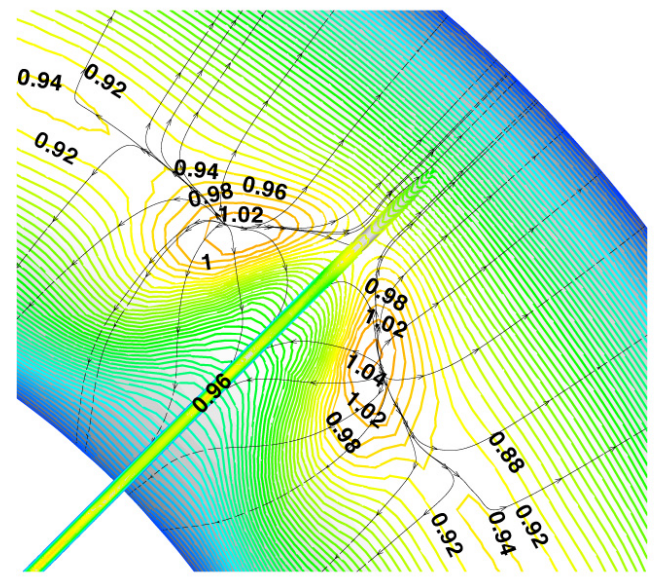

(c) Pressure

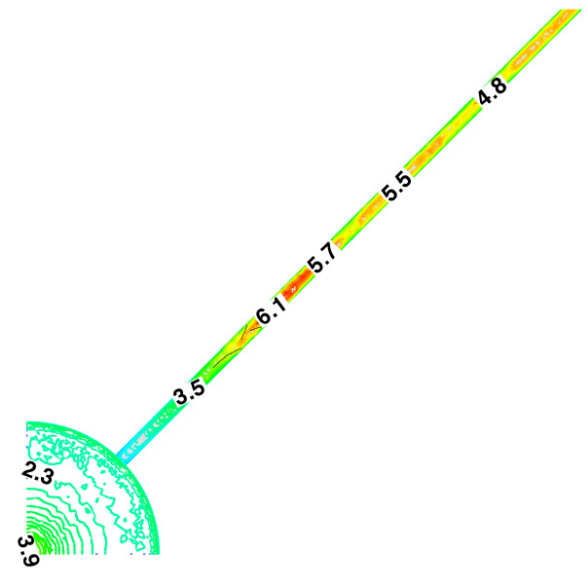

(b) Heating

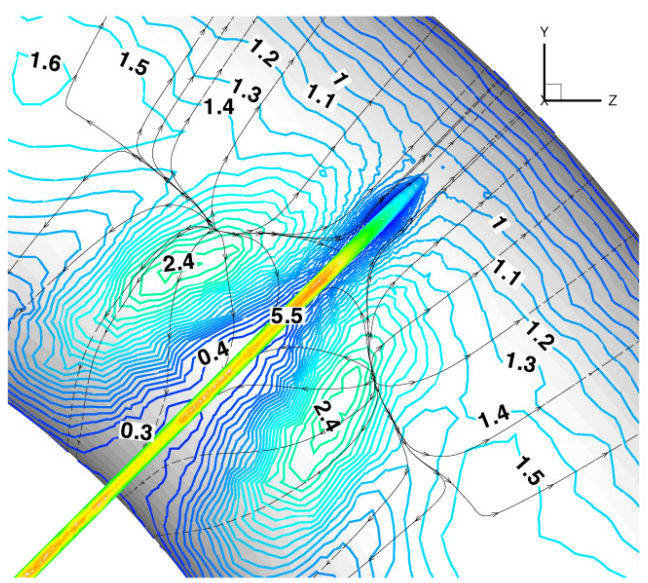

(d) Heating

Figure 8. Detail front view of surface pressure and heating.

limiting expression for a thin tether predicts a heating rate equal to $3.9 \mathrm{~W} / \mathrm{cm}^{2}$ for the Titan entry case $\left(V_{\infty}=8550 \mathrm{~m} / \mathrm{s}\right.$ and $\left.\rho_{\infty}=1.910^{-7} \mathrm{~kg} / \mathrm{m}^{3}\right)$ analyzed with FUN3D. However, the FUN3D nominal result for attachment line heating on the tether $\left(\approx 5 \mathrm{~W} / \mathrm{cm}^{2}\right)$ exceeds this limiting value. The FUN3D analysis is expected to be higher than the DS2V analysis because Navier-Stokes simulations tend to produce sharper gradients in the transitional flow regime than DSMC and a lower wall temperature and no slip conditions are applied to the FUN3D simulation.

The vibrational temperatures over a 0.50 meter compressive tether (Fig. 11) should be contrasted to the equivalent solution for the $1 \mathrm{~cm}$ diameter tensile tether. In this case, the peak vibrational temperature is still significantly below the kinetic temperature but the peak occurs on the leading edge. A radiative equilibrium wall boundary condition was applied to evaluate surface temperature for the compressive tether. The peak surface temperature in this case is approximately $800 \mathrm{~K}$ with peak heating rate at $2 \mathrm{~W} / \mathrm{cm}^{2}$. Embedding the tensile load carrying members within the larger diameter compressive tether provides some relief for tensile tether design by reducing peak tether temperatures. The compressive tether, with Knudsen number 0.064, appears in the middle of the bridging function domain between free molecular and continuum flow. The tensile tether, with Knudsen number 3.2 for $1 \mathrm{~cm}$ diameter, occurs near the upper end of the bridging function domain at the free molecular limit. 

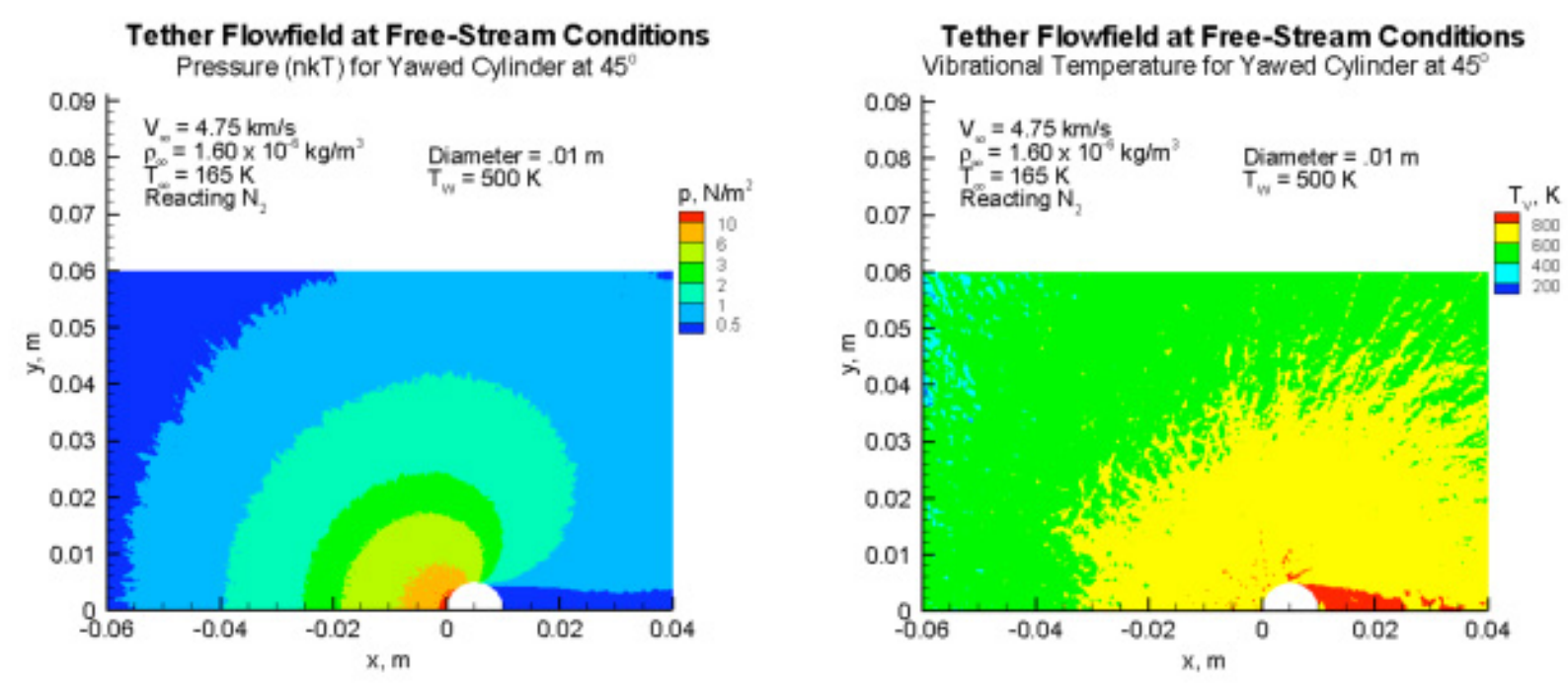

Figure 9. Pressure and temperature contours over $1 \mathrm{~cm}$ diameter tensile tether.
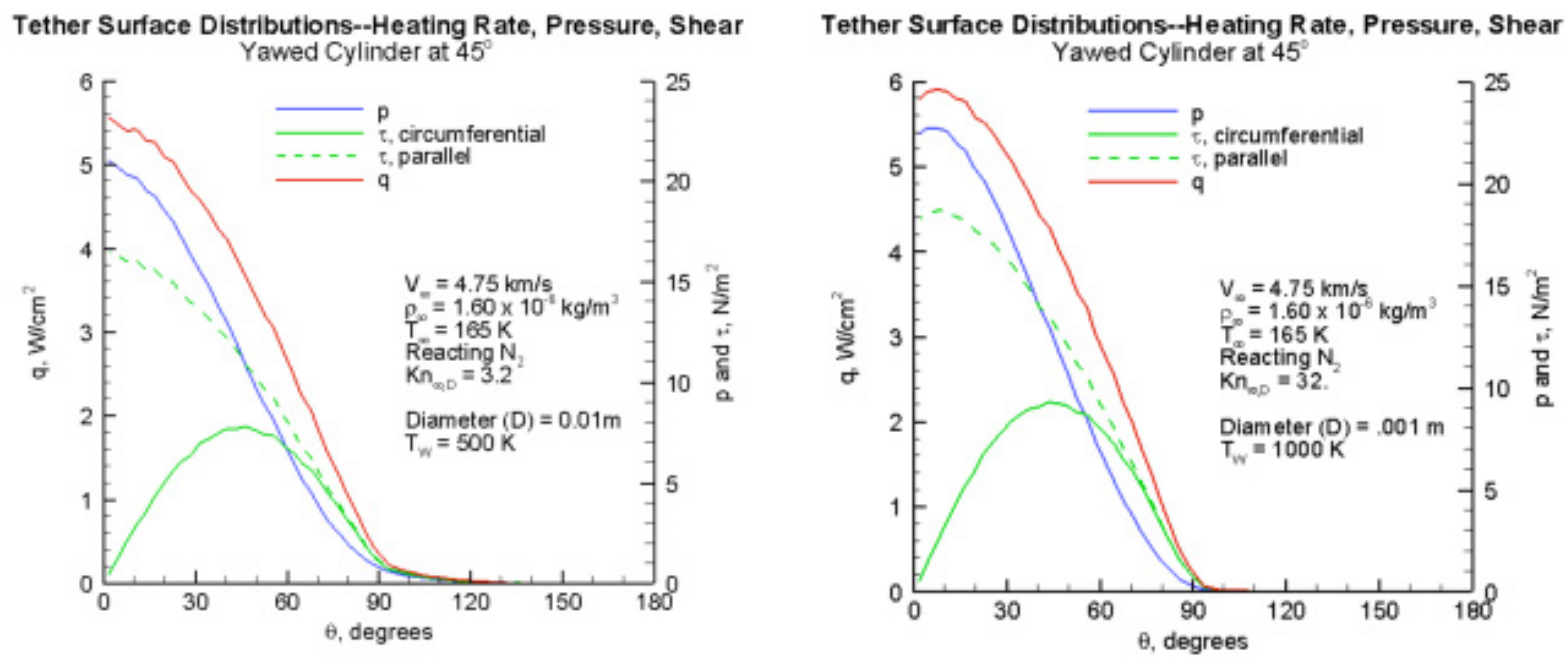

Figure 10. Heating, pressure, and shear distributions over $1 \mathrm{~cm}$ and $1 \mathrm{~mm}$ diameter tensile tethers. 
Tether Flowfield at Free-Stream Conditions

Overall KineticT emperature for Yawed Cylinder at $45^{\circ}$

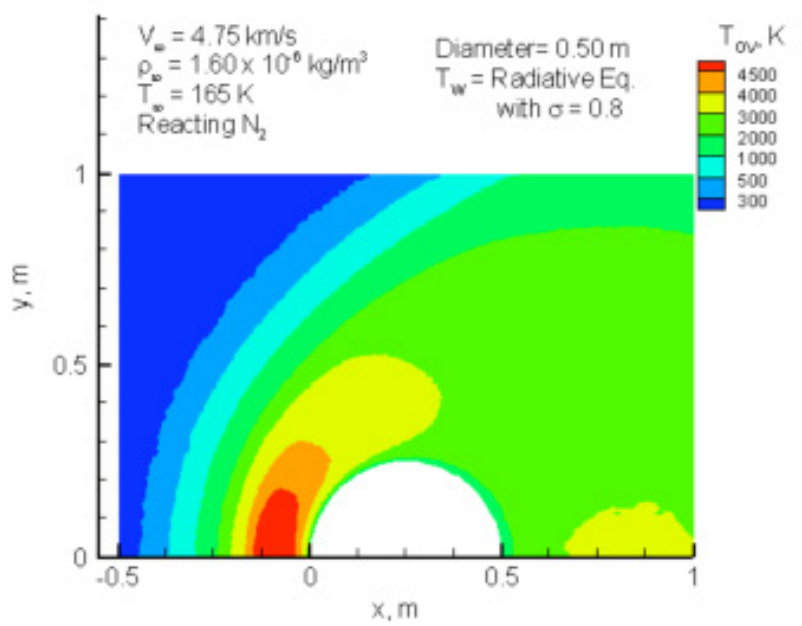

Tether Flowfield at Free-Stream Conditions

Vibrational Temperature for Yawed Cylinder at $45^{\circ}$

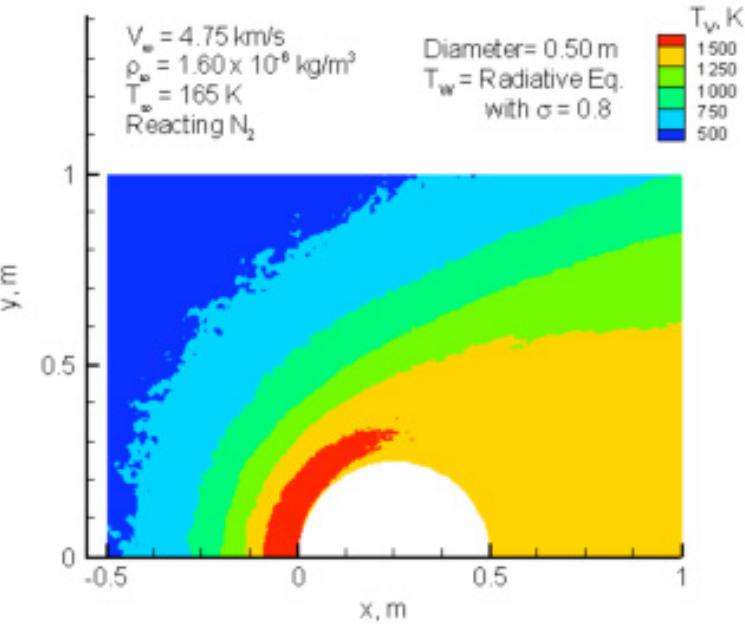

Figure 11. Pressure and temperature contours over $50 \mathrm{~cm}$ diameter compressive tether.
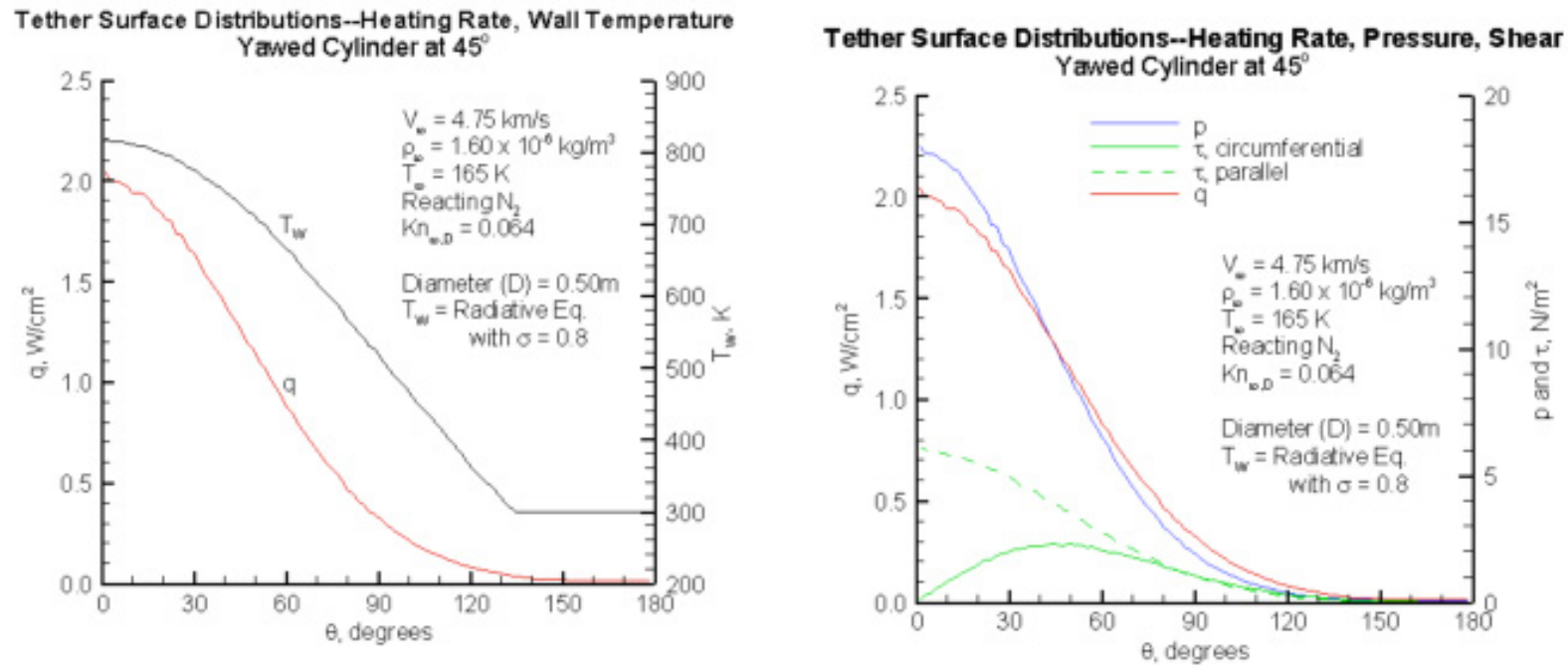

Figure 12. Heating, pressure, and shear distributions over $50 \mathrm{~cm}$ diameter tensile tethers. 


\section{Unsteady Flow}

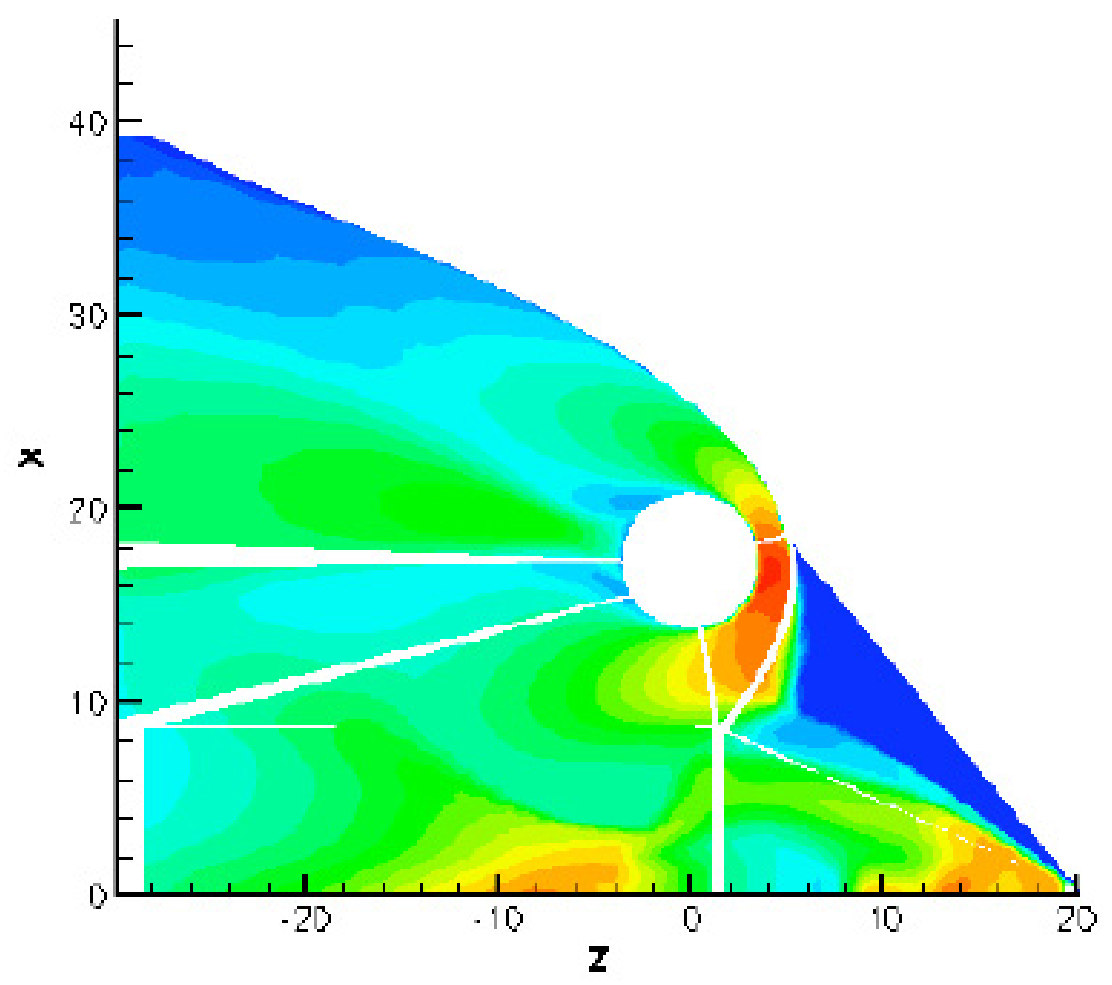

Figure 13. Single frame of CFD simulation for entry condition at Titan with weak unsteady interaction in the wake of the spacecraft.

\section{A. Flight Simulation}

The interaction of the towing spacecraft wake with the Mach disc in the core of the toroidal ballute is known to produce unsteady flow in the limit of inviscid flow (high Reynolds number). High altitude, viscous simulations do not indicate any significant unsteadiness in the vicinity of the peak heating trajectory point. As the system descends lower into the atmosphere, Reynolds number continues to increase and the potential for unsteady flow is enhanced. Time accurate simulations (first-order temporal, second-order spacial) indicate that unsteadiness is encountered at a maximum Reynolds number condition just prior to release of the ballute. Conditions for the simulation of hypersonic flow over a toroidal ballute just prior to ballute release in a nitrogen atmosphere are: $V_{\infty}=2530 \mathrm{~m} / \mathrm{s}, \rho_{\infty}=4.5310^{-6} \mathrm{~kg} / \mathrm{m}^{3}, M_{\infty}=9.75$, and $R e_{\infty}=12001 / \mathrm{m}$. A single frame from the simulation is presented in Fig. 13. The extent of motion is much less severe than that encountered in the inviscid limit. The significance of this result is that unsteady motions can ensue within nominal towed ballute trajectories and one must verify that the extent of such motion is not so severe as to compromise the system stability or integrity. In this worst case scenario, the extent of unsteady motion causes only small perturbations on the surface of the toroid. Backwash of flow over the base of the spacecraft is observed; consequently, one must confirm that the heating to spacecraft components exposed on the base is within acceptable limits or one must protect the base with a shroud.

\section{B. Simulation of Ground-Based Experiment}

The flight simulation of Fig. 13 indicated that a moderate level of unsteadiness is predicted prior to ballute release. An obvious concern is that neither the prediction of the onset of unsteady flow nor the extent of unsteady flow has been verified in any related context. A series of wind tunnel tests were conducted at 


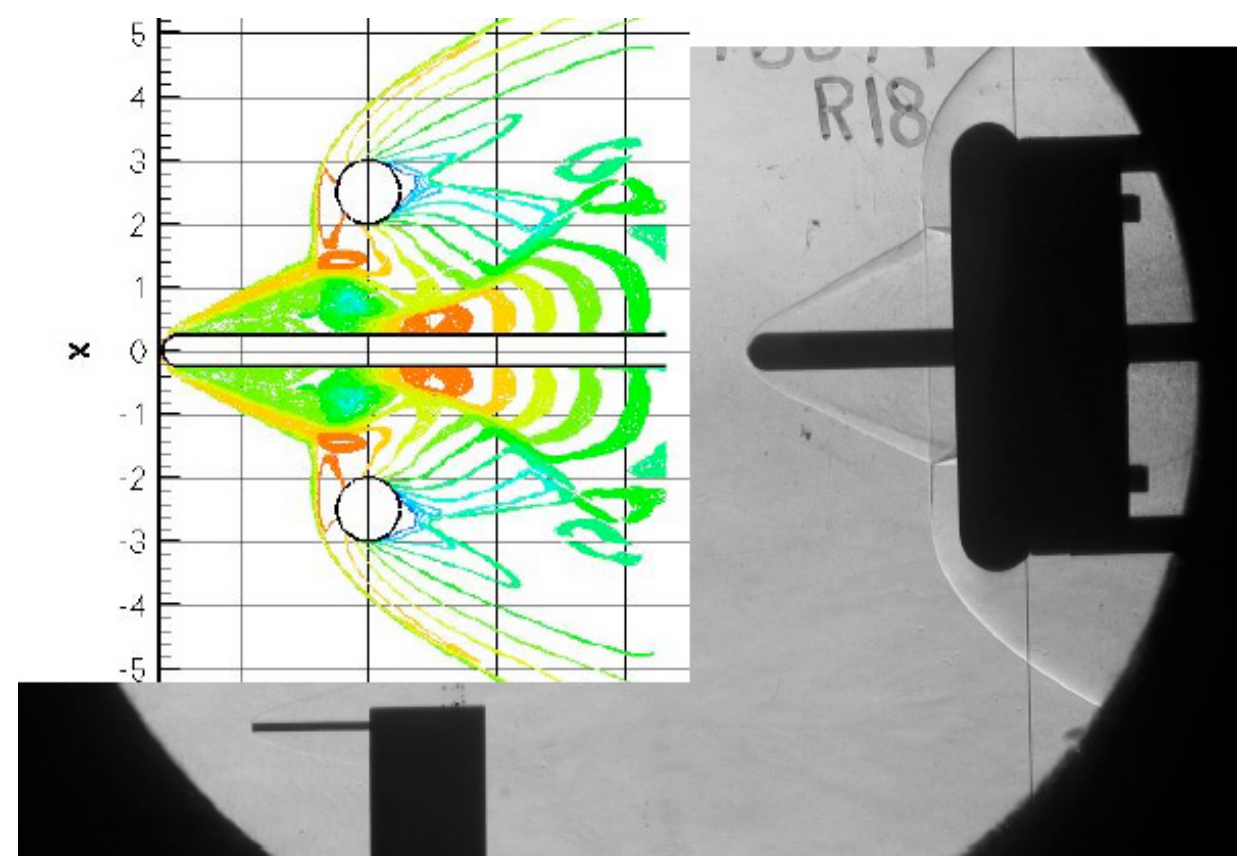

Figure 14. Sclieren photo of run 18 condition with density contour plot from simulation. Fifty frames of the LAURA simulation are overlayed to define the extent of density variation at any point in the symmetry plane.

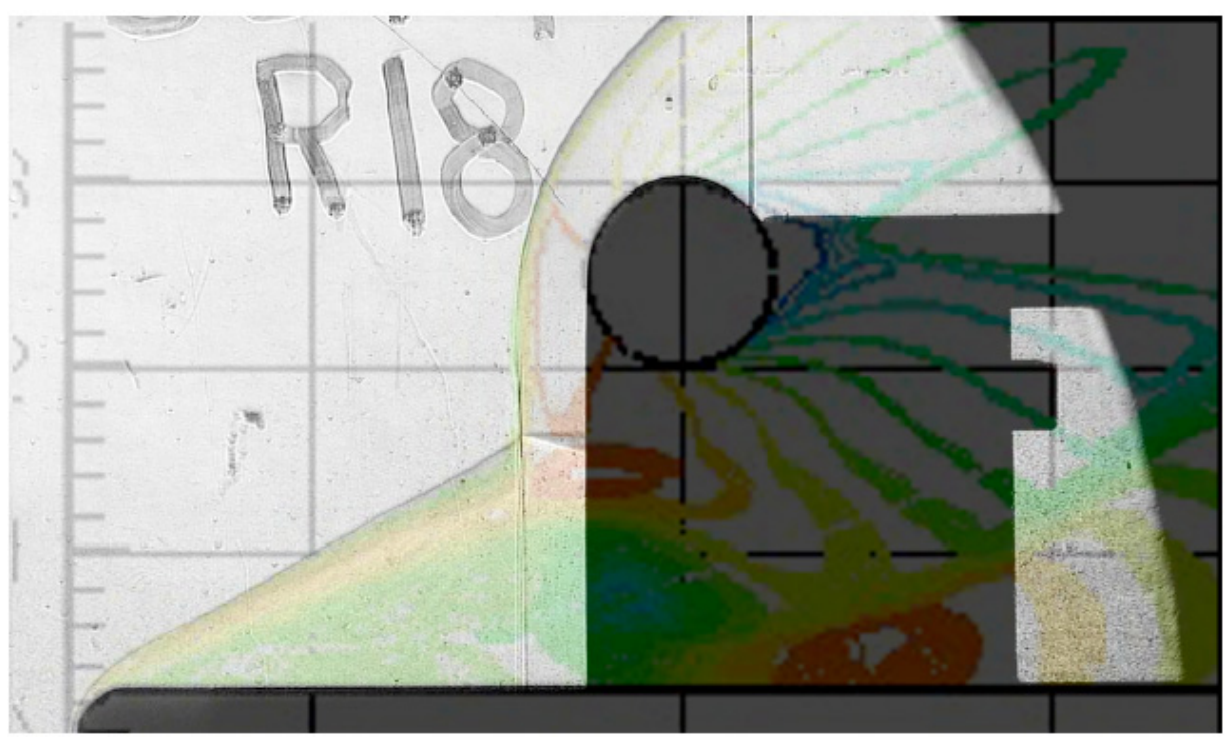

Figure 15. Sclieren photo of run 18 condition overlayed on contour plot from LAURA simulation. Shock standoff is accurately captured. The simulated shock interaction appears to be diffused relative to the schlieren due to an inability to adequately adapt grid to local structure.

Langley in Mach 6 air and CF4 to gather surface heating data (thermophosphor) and Schlieren video to observe unsteady motion. In the video frame in Fig. 14 a hemispherically capped cylinder is shown extending through the center of the toroid. The spacecraft (cylinder) diameter is 0.50 inches. The toroid diameter is 6 inches. It produces a bow shock which is directed just inside the inner boundary of the toroid. As the cylinder is pushed further upstream, unsteady motion ensues. As the cylinder is retracted the motion appears steady (at least with regard to images captured in the Schlieren). The dynamics of the wind tunnel case are somewhat different than the flight system in that the cylindrical sting is not present in flight. Nevertheless, 


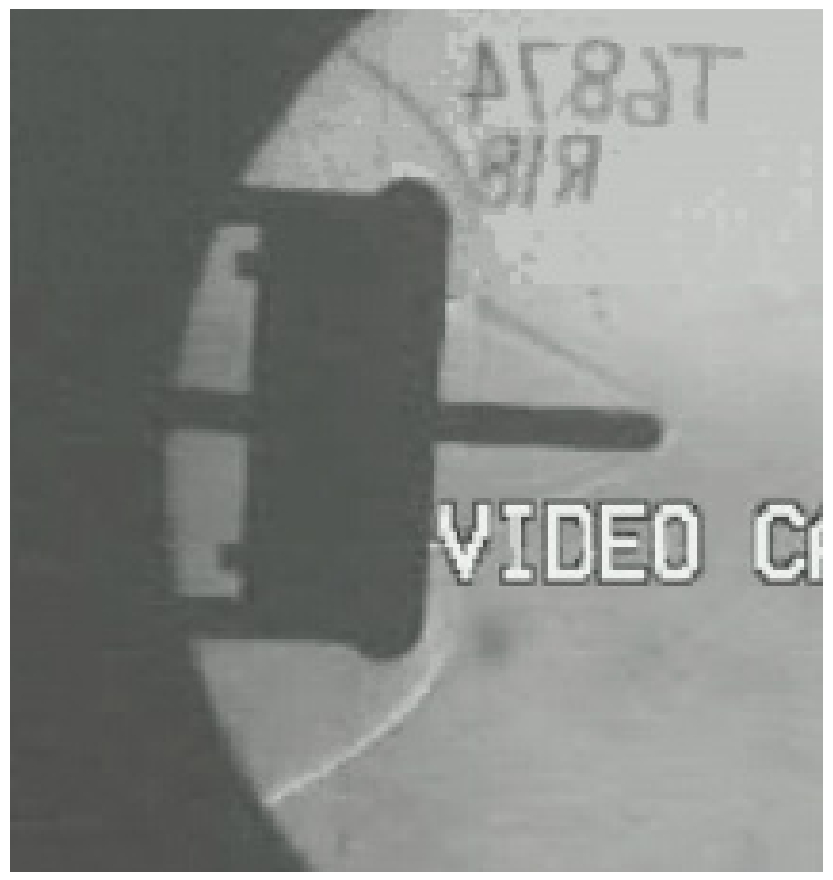

Figure 16. Single frame of Schlieren video for Run 18 with weakly unsteady flow (small amplitude excursions).

the same behavior of the recirculating flow moving forward due to the compression in the core of the toroid is inferred from the pulsing bow shock behind the nose of the cylinder. The color inset to the left of Fig. 14 is the first-order-time-accurate LAURA simulation of the experiment in Mach 6 air in which 50 frames (density contours) of the simulation are superposed. If flow is steady then contour lines overplot as a thin line. The extent of smearing of any line indicates the range of motion.

The experimental and computational images of Fig. 14 are overlayed in Fig. 15 to show excellent agreement of the shock front. Note in this case that the slight undulations of the bow shock above the cylinder indicate a low amplitude, unsteady response to the recirculating flow underneath.

Figure 16 is a single frame from a Schlieren video of Run 18 in the 20-Inch Mach 6 Air Tunnel at a Reynolds number of 1 million per foot. The distance from the center of the hemispherical nose to the midplane of the toroid is 3 inches. The video frame rate is not sufficiently high to resolve a single cycle of recirculating flow. Instead, it captures instantaneous (to exposure time) locations of the bow shock as it responds to the unsteady, separated flow beneath it. The observed range of motion in this case is characterised as incipient unsteady response. There is a slight fluttering with small wavelength visible in the bow shock image. This incipient case is considered an important test because we want to confirm the ability of LAURA to identify the presence and extent of unsteady motion.

A single frame from the simulation of Run 18 with LAURA is presented in Fig. 17 showing density contours. In the movie, there is significant movement of the reflected shock off the cylinder which drives the recirculation flow back to the nose. These structures cannot be picked up in the Schlieren. The response of the bow shock over the cylinder is more subdued. The fluttering of the bow shock in the CFD simulation appears to be somewhat stronger than observed in the experiment. The key point here is that CFD in fact picked up an unsteady response when one is observed in the experiment. In contrast, Run 16 (not shown here) in which the cylinder was retracted another inch backward produced a flow which was steady in the Schlieren and steady in the CFD simulation. This agreement builds credibility for the first time that the available tools are at least able to discern unsteady motion when it occurs. The more complex task of validating the time accurate response is of less concern in that we want to avoid the unsteady motion in the flight system in the first place (or at least insure that the extent of unsteady motion does not compromise the flight system).

An example of a violently unsteady response which would compromise system integrity is evident in Run 20 in which the cylinder is pushed an additional inch forward (as compared to Run 18). In this configuration 


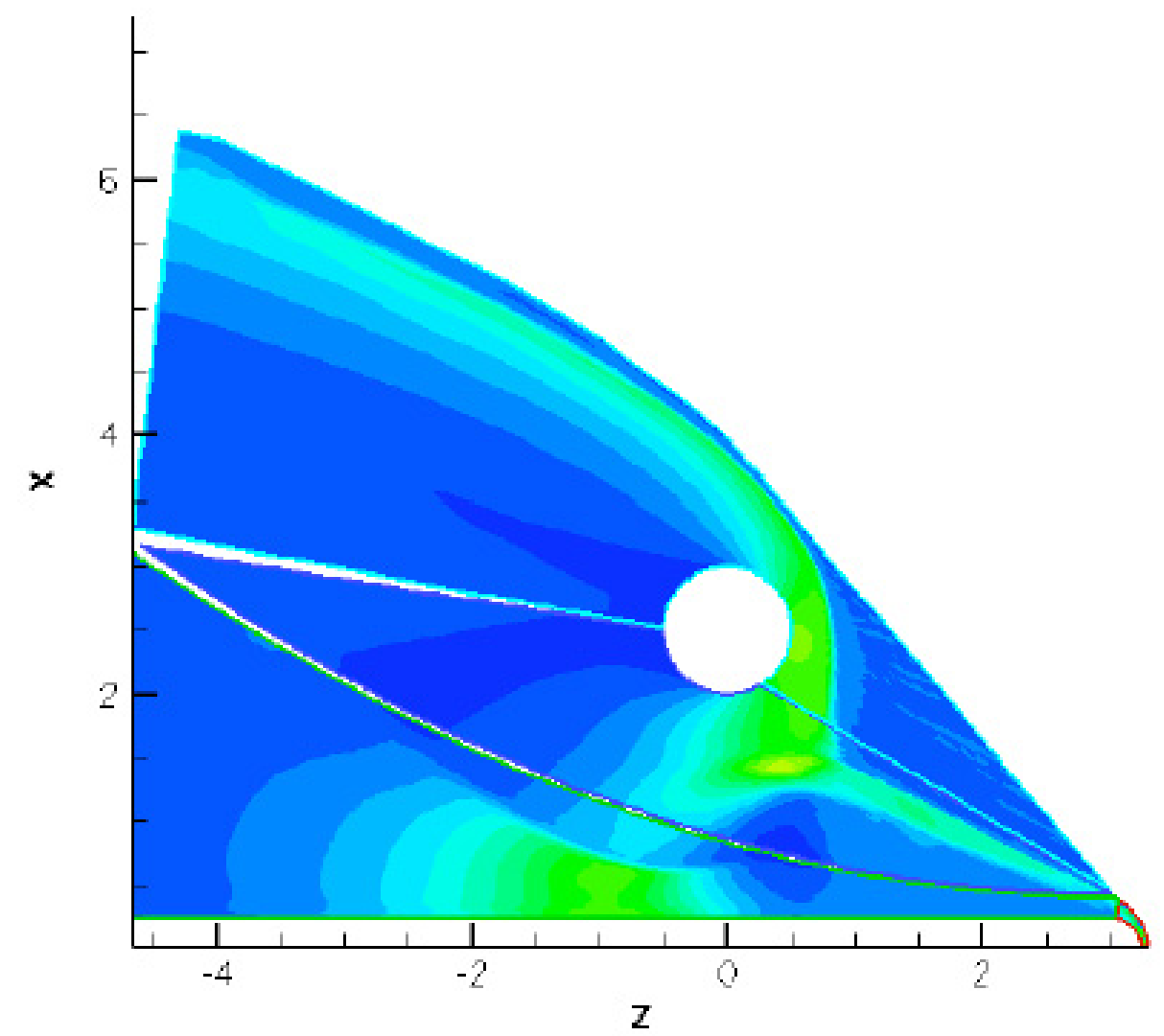

Figure 17. Single frame of CFD simulation for Run 18 with weakly unsteady flow (small amplitude excursions). The magnitude of oscillations is captured.

(Fig. 18) the bow shock impinges on the toroid. A more complex wave pattern reflects off the cylinder in the core of the toroid, and a massively unsteady response ensues. The bow shock off the cylindrical nose moves from the inner to outer edge of the toroid - a situation totally unacceptable in flight from both a heating and system stability perspective. The unsteady simulation of Run 20 (Fig. 19) shows an equivalent amplitude of motion as the experiment. The bow shock off the spherically capped cylinder swings from the inside to outside edge of the trailing toroid.

\section{Conclusions}

Two challenging aspects of aerothermal simulation of towed ballutes are studied. The first challenge, simulation of a complete system including inflatable tethers and a trailing toroidal ballute, is addressed using the unstructured-grid, Navier-Stokes solver FUN3D. Auxiliary simulations of a semi-infinite cylinder using the rarefied flow, Direct Simulation Monte Carlo solver, DS2V, provide additional insight into limiting behavior of the aerothermal environment around tethers directly exposed to the free stream. Simulations reveal pressures higher than stagnation and corresponding large heating rates on the tether as it emerges from the spacecraft base flow and passes through the spacecraft bow shock. A staged compression through the spacecraft bow shock and then tether shock produce pressures slightly higher than stagnation. The small tether radius serves to amplify the heating in this interaction which is about $20 \%$ greater than nominal heating values along the tether attachment line. The heating rate on the ballute surface is approximately $50 \%$ larger than nominal rates on the attachment line away from the tether where the footprint of the tether shock impinges on the ballute surface. A more benign interaction may be expected if the tether were to be attached from a more outboard location on the spacecraft and connected to the inboard tangency point on the toroid. This setup will reduce the inclination angle of the tether relative to the free stream and will divert 


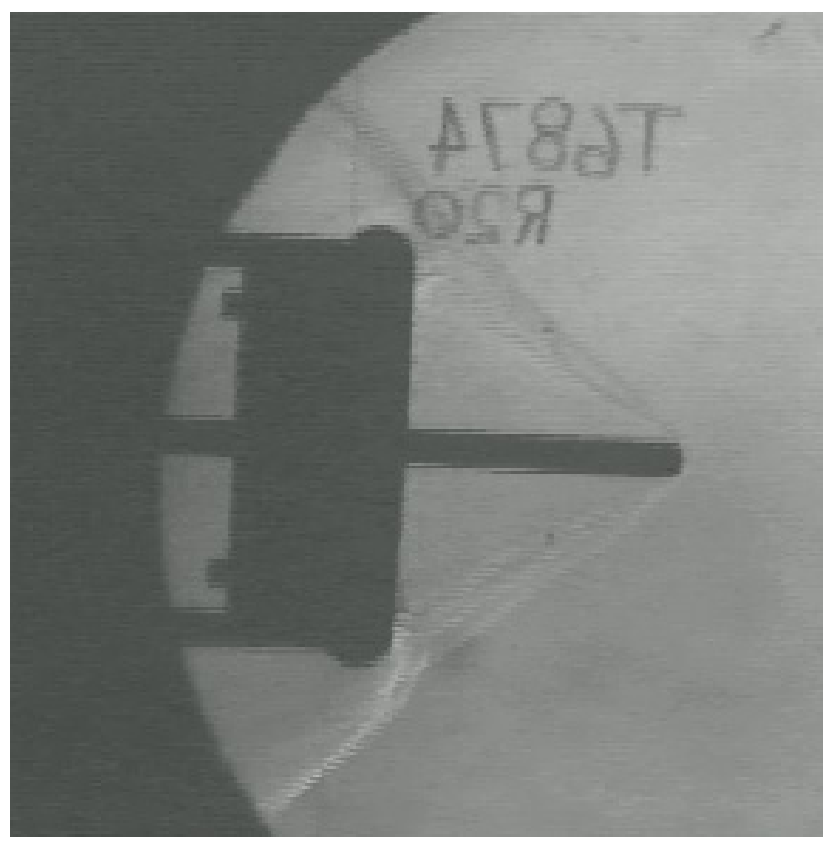

Figure 18. Single frame of Schlieren video for Run 20 with strong unsteady flow (large amplitude excursions).

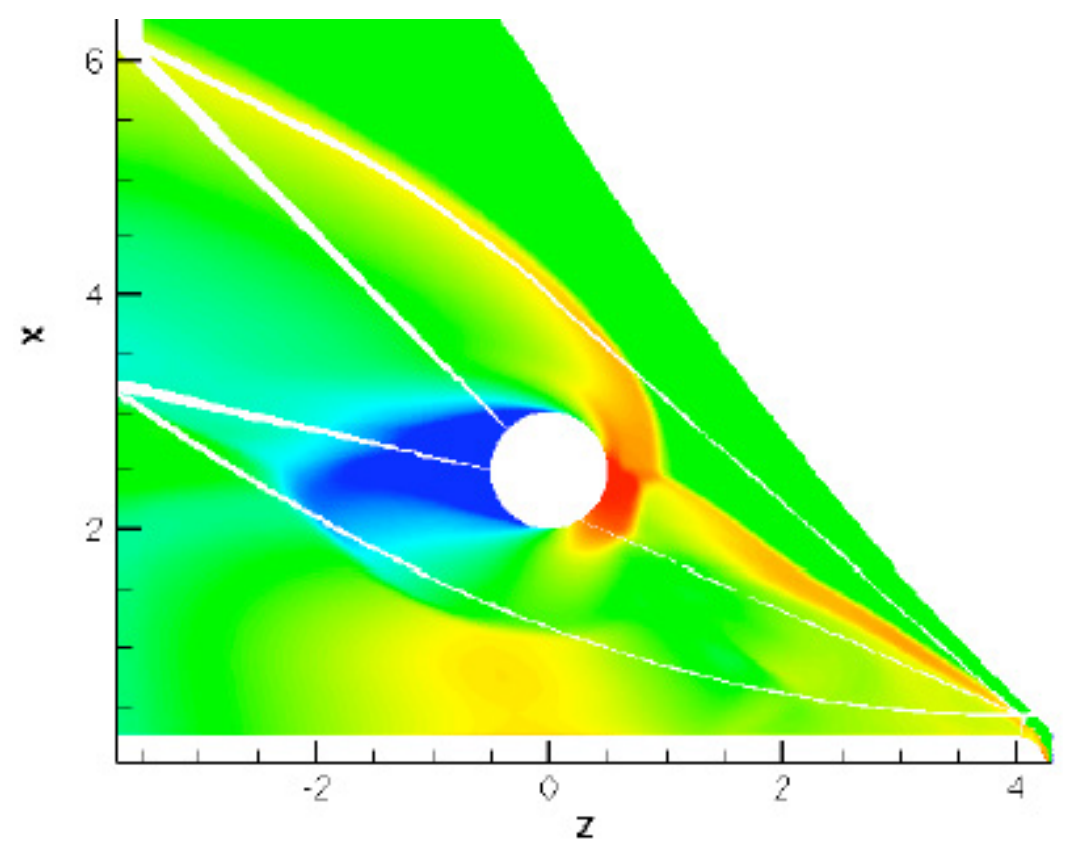

Figure 19. Single frame of CFD simulation for Run 20 with strong unsteady flow (large amplitude excursions). The magnitude of oscillations is qualitatively captured.

the tether wake away from the ballute surface which serve to reduce heating levels. A possible downside of such a configuration is the pinching of the flow between the tether and ballute (and associated heating increase) as it expands towards the wake.

A series of DSMC simulations have been executed on an idealized tether, treated as an infinite yawed cylinder in a uniform, hypersonic flow. The cylinder is inclined to the flow at 45 degrees. A limiting correlation for heating on a thin, infinite yawed cylinder in the transitional to free molecular regime is 
identified for the case of a non-catalytic surface in nitrogen.

The second challenge studied herein addresses time-accurate simulation to detect the onset of unsteady flow interactions as a function of geometry and Reynolds number. Video of unsteady interactions measured in the Langley Aerothermodynamic Laboratory 20-Inch Mach 6 Air Tunnel and CFD simulations using the structured grid, Navier-Stokes solver LAURA are compared for flow over a rigid spacecraft - sting - toroid system. The experimental data provides qualitative information on the amplitude and onset of unsteady motion which is captured in the numerical simulations. The presence of severe unsteady fluid - structure interactions is undesirable and numerical simulation must be able to predict the onset of such motion. Comparisons with this data set demonstrate that a first-order accurate in time Navier-Stokes simulation is able to predict the onset of unsteady interactions feeding back to the bow shock.

\section{Acknowledgements}

This work has been funded through In-Space Propulsion (ISP) by NASA Office of Space Science under contract NAS8-02130 and represents part of an overall effort led by Ball Aerospace, including ourselves at Langley as well as partners at ILC Dover, Jet Propulsion Laboratory, Johnson Space Center, Georgia Tech, and the University of Virginia to raise the technology readiness level of large, high altitude, inflatable drag devices. The authors thank Kevin Miller and Jim Mascarelli at Ball for their leadership and technical interactions.

\section{References}

${ }^{1}$ Hall, J. L., "A Review of Ballute Technology for Planetary Aerocapture," IAA Paper L-1112, May 2000. 2001.

${ }^{2}$ Hall, J. L. and Le, A., "Aerocapture Trajectories for Spacecraft with Large Towed Ballutes," AAS Paper 01-235, Feb.

${ }^{3}$ Masciarelli, J. P., Lin, J. K., Ware, J. S., Rohrschneider, R. R., Braun, R. D., Bartels, R. E., Moses, R. W., and Hall, J. L., "Ultra Lightweight Ballutes for Return to Earth from the Moon ," AIAA Paper 2006-1698, May 2006.

${ }^{4}$ Masciarelli, J. P., Miller, K., Stein, J., Ware, J., Lawles, D., Westhelle, C., Gnoffo, P., Buck, G., and McDaniel, J., "Fly Higher, Fly Lighter, Fly Cooler: Progress in Ultralightweight Ballute Technology Development," 53rd Joint Army-Navy-NASAAir Force (JANNAF) Propulsion Meeting, Monterey, CA, Dec. 2005.

${ }^{5}$ Gnoffo, P. A. and Anderson, B. P., "Computational Analysis of Towed Ballute Interactions," AIAA Paper 2002-2997, June 2002.

${ }^{6}$ LeBeau, G. J., "A User Guide for the DSMC Analysis Code (DAC) Software for Simulating Rarefied Gas Dynamic Environments," Revision DAC97-G 4674, 2002.

${ }^{7}$ Cheatwood, F. M. and Gnoffo, P. A., "User's Manual for the Langley Aerothermodynamic Upwind Relaxation Algorithm (LAURA)," NASA TM 4674, April 1996.

${ }^{8}$ Gnoffo, P. A., Gupta, R. N., and Shinn, J. L., "Conservation Equations and Physical Models for Hypersonic Air Flows in Thermal and Chemical Nonequilibrium," NASA TP 2867, Feb. 1989.

${ }^{9}$ Hornung, H. G., "Hypersonic Flow over Bodies in Tandem," Shock Waves, Vol. 11, No. 6, 2002, pp. 441-445.

${ }^{10}$ Anderson, W. K. and Bonhaus, D. L., "An Implicit Upwind Algorithm for Computing Turbulent Flows on Unstructured Grids," Comp. and Fluids, Vol. 23, No. 1, Jan. 1994, pp. 1-21.

${ }^{11}$ White, J. A. and Morrison, J. H., "A Pseudo-Temporal Multi-Grid Relaxation Scheme for Solving the Parabolized Navier-Stokes Equations," AIAA Paper 99-3360, June 1999

${ }^{12}$ Roe, P. L., "Approximate Riemann Solvers, Parameter Vectors, and Difference Schemes," J. Comput. Phys., Vol. 43, No. 2, Oct. 1981, pp. 357-372.

${ }^{13}$ Yee, H. C., "On Symmetric and Upwind TVD Schemes," NASA TM 88325, 1986.

${ }^{14}$ Gnoffo, P. A., "On the Numerical Convergence to Steady State of Hypersonic Flows Over Bodies With Concavities," West East High Speed Flow Field Conference, Marseilles, France, 2002.

${ }^{15}$ Mitterer, K., Mitcheltree, R., and Gnoffo, P., "Application of Program LAURA to Perfect Gas Shock Tube Flows: A Parametric Study," NASA TM 104190, January 1992.

${ }^{16}$ Bird, G. A., Molecular Gas Dynamics and the Direct Simulation of Gas Flows, Clarendon Press, Oxford, 1994.

${ }^{17}$ Bird, G. A., Visual DSMC Program for Two-Dimensional and Axially Symmetric Flows, The DS2V Program User's Guide, Version 2.1, 2003.

${ }^{18}$ Borgnakke, C. and Larsen, P. S., "Statistical Collision Model for Monte Carlo Simulation of Polyatomic Gas Mixture," J. Comput. Phys., Vol. 18, No. 4, 1975, pp. 405-420.

${ }^{19}$ Gnoffo, P. A., "Computational Fluid Dynamics Technology for Hypersonic Applications," AIAA Paper 2003-3259, July 2005.

${ }^{20}$ Gnoffo, P. A. and White, J. A., "Computational Aerothermodynamic Simulation Issues on Unstructured Grids," AIAA Paper 2004-2371, June 2004.

${ }^{21}$ Moss, J. N., "DSMC Simulations of Ballute Aerothermodynamics Under Hypersonic Rarefied Conditions," AIAA Paper 06-4949, June 2005. 